\title{
COMMERCIAL-OFF-THE-SHELF SOFTWARE BECOMES MISSION-CRITICAL TO SUCCESS AND COST-EFFECTIVE SPACE MISSIONS
}

\author{
Paul L. GRAZIANI
}

\begin{abstract}
Governmental space budgets are tightening. The era of huge space programs with J virtually unlimited budgets is over. Meanwhile, the markets for commercial services delivered by satellite continue to grow at $17 \%$ CAGR, ${ }^{1}$ driven by increased access to launch services, lower-cost satellites, and global-scale business opportunities. Competition, though, means that cost-effectiveness is more critical to the success of a mission than ever.

Many organizations can no longer afford to develop mission-specific software inhouse. Commercial satellite software that can be used for multiple missions is playing a growing, cost-saving role in enabling not only successful but economical space activities. Commercial off-the-shelf (COTS) software that is stable, accurate, and well-supported is key in all phases of a satellite's life-from pre-launch analyses to ground support through to graveyarding.
\end{abstract}

\section{Software Development Costs}

Historically, satellite programs have been funded to develop custom software solutions from scratch. It has been identified that software development is the largest cost-risk factor during the development of a new satellite system. This is driven by two factors:

- $\quad$ Lines of code underestimated

- $\quad$ Technological challenges - programmer productivity decreases

The chart below identifies four large satellite programs which all significantly underestimated the scope of their software requirements which resulted in significant cost overruns and failure to meet delivery schedule. 


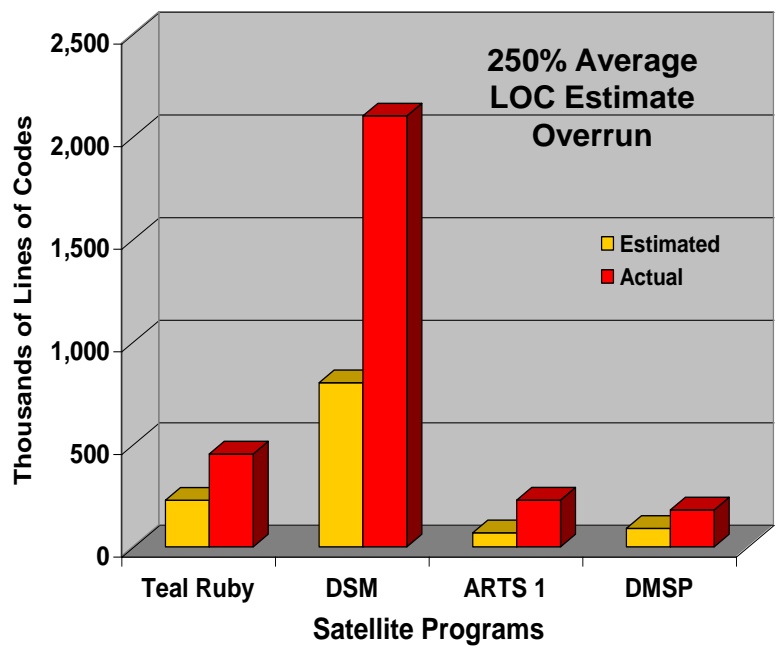

Typical satellite programs have shown a Line-Of-Code (LOC) overrun of approximately $2.5 \mathrm{X}$ - to compound the software development risk, programmer productivity typically falls to about $28 \%$ of estimated rate $(3.5 \mathrm{X}$ slower than estimated). Due to competitive forces, aerospace contractors are forced to bid/estimate programmer productivity at a rate of 300 LOC per month; however, the average productivity turns out to be 85 LOC because of unforeseen technological challenges as well as unrealistic expectations.

\section{Software Cost-Growth Factor}

- $\quad 250 \%$ growth in LOC estimate

- $350 \%$ slip in productivity

- $250 \%$ X $350 \%$

$880 \%$ cost growth in satellite software projects

Another unpredictable cost associated with in-house or built-from-scratch software solutions is that follow-on maintenance of the software project is often not considered within the initial funding of the program, key developers may leave the project, or unneeded contractors may become entrenched into the program office.

COTS software has proven that it can meet the unique needs of each space program but not require the years to create and millions of dollars to produce. COTS software has also proven that it has the technical depth and diversity of capabilities to handle risky situations where new ground is broken, both figuratively and literally. This 
increasing reliance on COTS solutions demands better and more robust products that can address a wide variety of analysis and planning needs. Customers are looking for an economical solution that remains technically accurate and easy to use.

\section{The IMACCS Project}

NASA's Mission Operations and Data Systems Directorate (MO\&DSD) created the Reusable Network Architecture Interoperable Space Science, Analysis, Navigation, and Control Environment (RENAISSANCE) project to re-engineer the process used to build and operate spacecraft ground support systems. The primary goal of the RENAISSANCE project was to achieve cost-effective and flexible development and operations based on workstation/LAN/file server technology and reusable hardware and software building blocks.

The benefit of using COTS software in the RENAISSANCE effort was the construction of reusable, generic, software "building blocks" based on legacy software. In theory, new missions could then select among these building blocks to build systems in an object-oriented manner.

CSC, a contractor to NASA, suggested that an approach emphasizing the use of COTS products rather than software developed in-house would be far more cost effective. Although it was commonly believed that the COTS market had yet to reach sufficient maturity for most ground support functions, some key NASA personnel backed CSC's proposal. As a result, the Integrated Monitoring, Analysis and Control COTS System (IMACCS) project was approved.

Based in part on prior positive experiences with COTS satellite analysis software systems, CSC was confident that COTS systems could be utilized to produce a robust system that could meet or exceed the current ground support system in terms of functionality, implementation time, and implementation costs. The IMACCS challenge was to assemble a COTS-based system that could mirror current ground support, from data decommutation and conversion through spacecraft monitoring and commanding. IMACCS would also be responsible for off-line activities such as orbit and attitude determination and predictive products.

To demonstrate the feasibility of a COTS system, CSC was tasked to create a proofof-concept system that performed all current ground support tasks. With a 90-day turnaround time constraint, CSC chose the Solar Anomalous Magnetospheric Particle Explorer (SAMPEX) satellite as the test vehicle for the proof of concept.

SAMPEX studies the energy, composition, and charge states of particles. It also closely monitors the magnetospheric particle populations that plunge occasionally into the middle atmosphere of the Earth, thereby ionizing neutral gases and altering 
the atmospheric chemistry. A key part of SAMPEX is to use the magnetic field of the earth as an essential component of the measurement strategy. The Earth's magnetic field is used as a giant spectrometer to separate different energies and charge states of particles as SAMPEX executes its near polar orbit. SAMPEX is thus a very versatile mission capable of scientific investigations in astrophysics, solar physics, magnetospheric physics and atmospheric physics. Figure 1 is a picture of the SAMPEX satellite orbiting Earth.

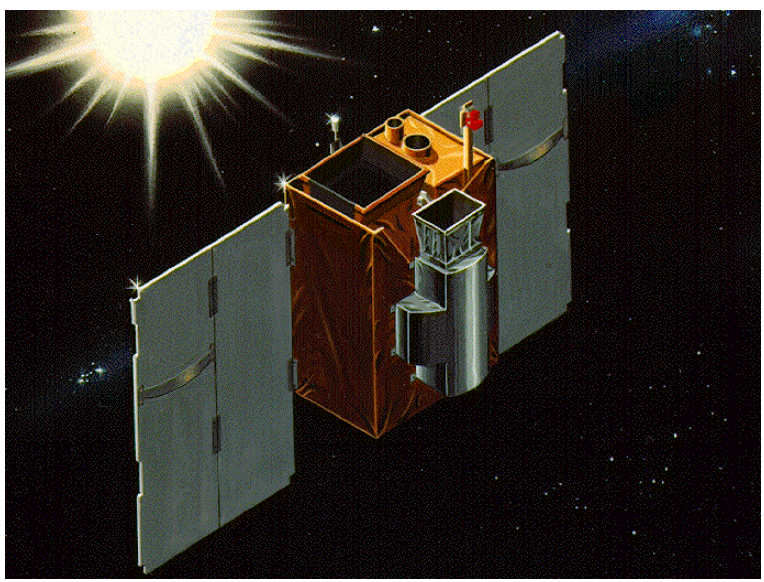

Figure 1: SAMPEX Satellite

Because SAMPEX is a relatively small program and is currently operational, it was ideal for the project. In addition, SAMPEX produces telemetry data in CCSDC format, a relatively new format that is fast becoming popular. The IMACCS project consists of several COTS systems. Figure 2 summarizes the IMACCS structure

IMACCS used the COTS software to perform orbit determination and propagate ephemeris. One module was used to accelerate access determination and group ground stations together for access reporting purposes. Other tasks performed by the IMACCS system included predicting potential ground station contact times, lighting times, times of passage through the South Atlantic Anomaly and other electron contamination regions, and node crossings. With this software, these prediction exercises could be easily viewed in the $2 \mathrm{D}$ map window as ground and/or orbital tracks. The software was also used to predict the position and velocity data delivered to the ground stations and control centers for uplink to the spacecraft. 


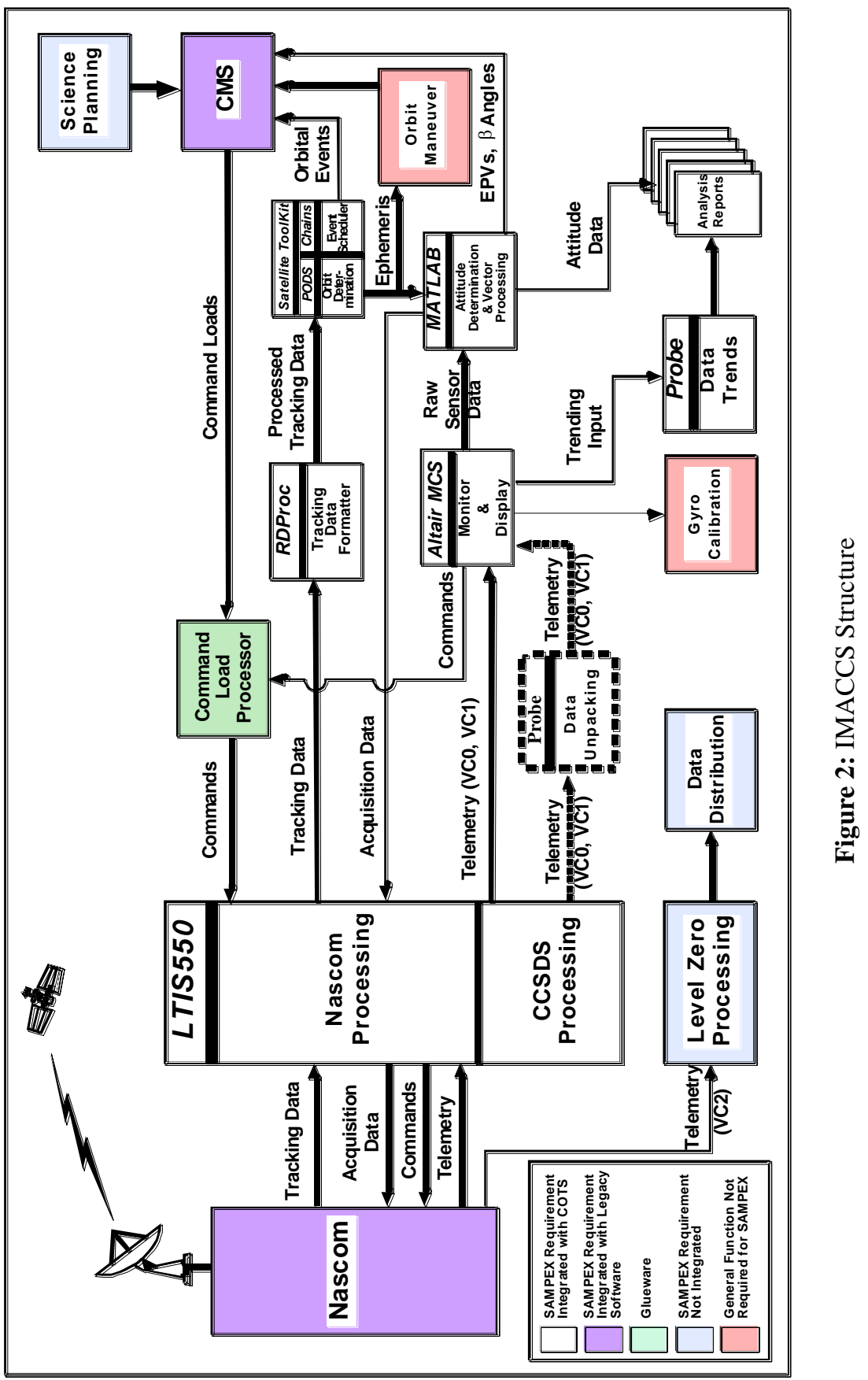




\section{Defining Regions of Interest}

The South Atlantic Anomaly is a variation in the Earth's magnetic field that allows cosmic rays and charged particles to reach deeper into the atmosphere. This can interfere with communications among spacecraft and aircraft, and may also corrupt scientific data gathered by the instruments onboard. CSC created an Area Target to represent the approximate size and shape of the Anomaly's widest boundary so that passage times through the anomaly could be computed and delivered to the Flight Operations Team (FOT). Figure 3 shows the area target as represented in the software's map window.

Area targets were also defined to represent regions that may or may not be of use to the SAMPEX satellite. Based on this data, control center personnel could determine whether instrumentation could be turned off to conserve power when the satellite traveled over these regions.

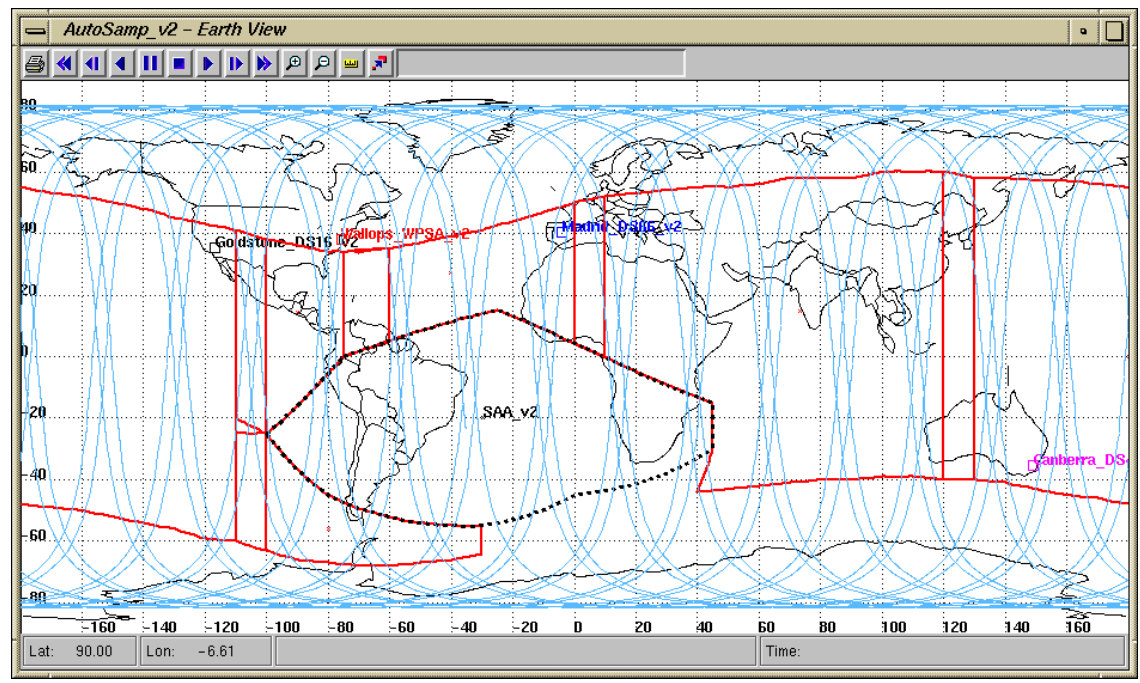

Figure 3: STK map window showing the South Atlantic Anomaly as well as the area targets defined for the SAMPEX mission

\section{Accurate Orbit Determination Made Easy}

To aid in the studies and measurements being conducted by the SAMPEX satellite, IMACCS was also designed to perform predictive orbit analyses. CSC chose add-on modules to accurately predict and propagate SAMPEX's orbit. Using measurements of SAMPEX's location over a period of time, CSC used a separate module to precisely determine the current orbit of the satellite. The module was then used to 
propagate the satellite's orbit into the near future. This information was then provided to the control center for mission planning and scheduling purposes.

CSC utilized another add-on module to simplify the process of scheduling and categorizing SAMPEX data, which allowed the user to link and/or group objects together - a powerful capability that enables the user to determine multiple accesses or multi-hop accesses. The user can create a constellation, which groups like objects together and establishes access constraints for the group of objects. Connectivity problems between multiple ground- and space-based objects or sets of objects can be rapidly analyzed with this feature set.

For the SAMPEX mission, CSC engineers were able to use the COTS package to determine station contact times for multiple ground stations simultaneously. The software was not limited to the number of ground stations or the location of the ground stations; the operators could even use Boolean operators to define connectivity requirements and priorities. Using this contact information, CSC could subsequently determine data collection times for each of the specific regions with unparalleled ease.

\section{Generating Reports}

IMACCS used several built-in report styles to document analysis results and orbital parameters. Reports documented the lighting times for the satellite as well as a variety of access times from the satellite to each facility, to all of the facilities (defined as a constellation), and to the South Atlantic anomaly (defined as an area target). In addition, reports summarizing azimuth, elevation and range data from the satellite to various facilities were generated.

The reports were then provided to the control centers as well as satellite schedulers for planning purposes.

One of the primary benefits of a COTS system is the fact that the technology has already been developed. The IMACCS prototype was developed in only 90 days. It was implemented on an operational satellite and successfully demonstrated in a short period of time. COTS products are typically more flexible than those developed inhouse on a mainframe or workstation. In addition, commercial tools are crossplatform compatible so that changing hardware requirements do not impact software performance. Most of the COTS software used in IMAACS runs on most UNIX platforms and is also available on the PC. STK data is saved in ASCII format for cross-platform compatibility and for easy export, if required. 


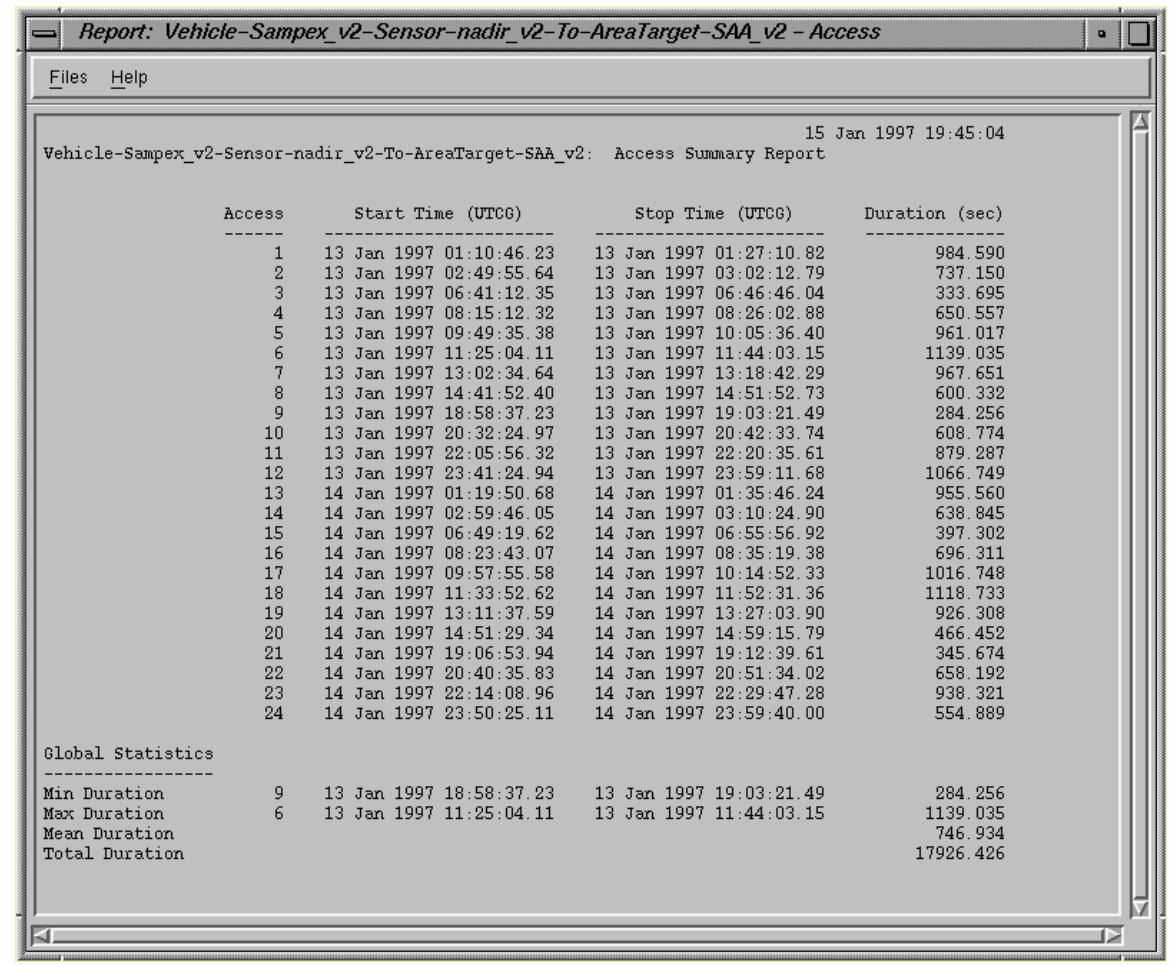

Figure 4: Sample report summarizing South Atlantic Anomaly "fly-through" times for SAMPEX

\section{Far Ultraviolet Spectroscopic Experiment (FUSE)}

As a value-added supplier of commercial-off-the-shelf (COTS)-based command and control solutions, Interface \& Control Systems, Inc. (ICS) has placed great emphasis on utilizing commercial products for satisfying specific, well-defined system requirements. ICS is under contract to the Johns Hopkins University as a team member of the FUSE Program. The FUSE satellite, a medium explorer mission, is designed to study the origin and evolution of the lightest elements - hydrogen and deuterium, and the forces and processes involved in the evolution of galaxies, stars, and planetary systems. ICS is responsible for the day-to-day management of the instrument and spacecraft activities within the Satellite Control Center, of which two integrated COTS products - SCL and STK- play an integral role. ICS realized that by using COTS products, their team would be guaranteed continued support for the entire mission. At a minimum the software met or exceeded the initial set of requirements, plus the team benefited from routine upgrades and the software's 
expandability and ability to interact with other products in the integrated software environment. COTS is used extensively for the operations of the FUSE satellite including:

- Real-time telemetry processing for on-orbit position and attitude visualization

- Generation of contact times

- Orbit propagation

- Generation of pointing angles for telescope operations

- Star tracking

The FUSE program was delivered at a reduced mission cost of $\$ 108$ million from the original budget of $\$ 254$ million, and the satellite was launched 2 years earlier than originally planned.

\section{The Future Is Now - Ellipso ${ }^{\mathrm{TM}}$}

The Ellipso satellite system is a constellation of communications satellites developed by Mobile Communications Holding, Inc. The Ellipso constellation is comprised of two subconstellations: Concordia and Borealis. The Concordia constellation consists of six operational satellites in circular equatorial orbits with an altitude of $8068 \mathrm{~km}$. The satellites in the Concordia constellation provide Earth ground coverage between the $55^{\circ}$ North and $55^{\circ}$ South parallels.

The second subconstellation, Borealis, consists of five operational satellites in each of two orbital planes. Both orbital planes are critically inclined and sun-synchronous. The Borealis constellation will not utilize the typical critical inclination of $63.4^{\circ} \mathrm{s}$ but will instead use the complementary retrograde inclination of $116.6^{\circ}$. Satellites in the first of the Borealis orbit planes have a local time of ascending node of noon, while those in the second orbit plane have a local time of ascending node of midnight. The Borealis ${ }^{\mathrm{TM}}$ orbits are eccentric with a perigee of $520 \mathrm{~km}$ and an apogee of $7846 \mathrm{~km}$, where perigee remains in the southern hemisphere. The satellites in the Borealis constellation provide additional coverage over the northern hemisphere.

The Ellipso satellite system, sometimes called Ellipsat, is the result of recent advances in communications technology with a highly innovative approach to using satellites. Its goal is to provide a simple, pragmatic and economical near-global telecommunications system. The FCC granted Ellipso a license on June 30, 1997. Once operational, it will provide combined position determination and mobile voice services using up to 17 satellites. Because of its unusual orbits, the Ellipso configuration is able to provide coverage to a large area with a small number of satellites. 


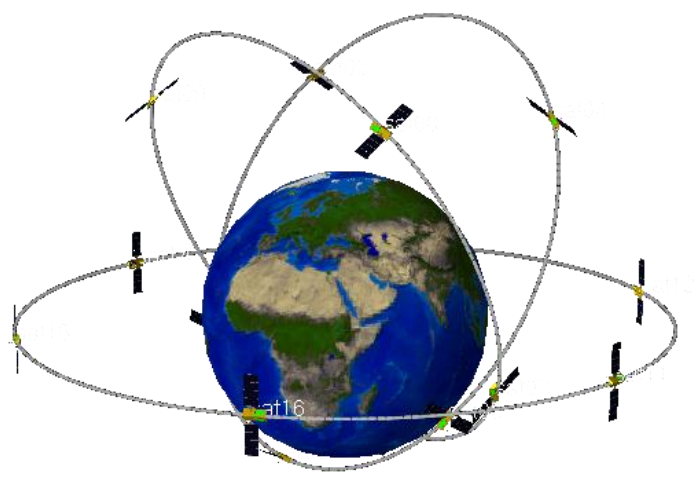

Figure 5: Graphical representation of the two constellations comprising the Ellipso satellite system

Spectrum Astro, Inc. performed analysis to determine the orbital stability and propellant budgets for the Ellipso system. To ensure that the launch itself is accomplished in the most economical manner while providing optimal satellite positioning, Mobile Communications Holding, Inc. contracted with Spectrum Astro to provide mission planning analyses. Spectrum Astro began designing the satellite and studying the constellation configuration as part of the Ellipso's conceptual orbit design phase.

Since the coverage characteristics of the system depended on the relative positions of the satellites and orbital planes, the effects of various orbit perturbations such as atmospheric drag, third body gravity, solar radiation pressure, and gravitational resonance on each subconstellation had to be well understood. A thorough understanding of the perturbations was especially important for the Borealis constellation since the planned time frame for launch during the year 2000 falls during a maximum in the solar activity cycle. The effect of atmospheric drag in the region near perigee of the Borealis orbits will be significantly increased due to the higher level of solar activity. As a result, more drag make-up maneuvers would be needed than would be necessary during times of lower solar activity.

The satellites that comprise the Ellipso system are designed to remain operational over a 7-year period. During this time, the satellites must maintain their orbits despite atmospheric drag at perigee. Spectrum Astro conducted an analysis of the long-term orbit evolution of the satellites. Because the Ellipso orbits are highly refined in shape and orientation, Spectrum Astro used STK and a number of add-on modules to model the satellites and their orbital planes. One of the goals of the Ellipso satellite system is to keep the orbits stable enough so that they pass over the same spot, at approximately the same time every day. Using commercial software, analysts are able 
to perform precise orbit predictions over the 7-year time period. Access reports are reviewed to identify orbit drift trends. Based on these trends, analysts can determine the best time for fuel burns to correct orbit drifts. Results enabled Spectrum Astro to determine the best way to maintain vehicle shape and orientation over the 7-year period by burning the least amount of fuel every few orbits.

This analysis phase is especially important for the Ellipso satellites because there is no historical data for this type of orbit. The need to maintain the constellation geometry over this time span means that the determination of accurate propellant budgets is critical. The goal of this effort is to determine a common sizing for the propulsion system of all satellites in the Ellipso constellation.

Command \& Control. The Ellipso satellite will be maintained using three ground command and control stations. Personnel at these stations will determine how often fuel burns should be performed to keep the satellites in orbit. They will also analyze and track the contact times between the satellites and the ground stations. To successfully fulfill their missions, the locations for the ground stations must be carefully chosen. In addition, the satellites themselves must have antennas that are positioned for optimal access.

To ensure that communications between satellites and ground stations are successful, Spectrum Astro conducted analyses to determine the number and position of antennas on the satellites as well as the optimal location for the three $\mathrm{C} \& \mathrm{C}$ ground stations. The Ellipso satellite system featured 61 different communications beams. To better define communications coverage, Spectrum Astro studied how often these beams overlap, which beams are used heavily, and how much coverage is provided on average.

In addition to mission planning and maintenance, analysts must also provide a plan for deorbiting, or graveyarding, the satellites at the end of their useful life. For the Concordia constellation, the orbital altitudes will most probably be raised so that the next set of satellites can be launched into orbit directly beneath the first set. Due to the elliptical nature of the Borealis constellation, operators will most likely perform a graveyard burn so that the satellites reenter the Earth's atmosphere in a few years. Graveyarding itself is dependent on the amount of fuel required for deorbiting and analysis tools such as STK can be used to determine reasonable opportunities for the deorbit of the satellites.

For the Ellipso program, Spectrum Astro used COTS software to perform mission analysis tasks. Spectrum Astro considers flexibility a valuable feature of COTS software. Analysts are able to export data for mission-unique analysis using other software packages that were developed in-house. They also import orbit trajectory data generated by mission-specific software into the COTS software for analysis. This 
flexibility allows Spectrum Astro to exploit all options for analysis without limiting the format and structure.

\section{Spacecraft Anomaly Resolution and Rescue}

The complexity of these space systems continues to increase as the demand for satellite services of various functionality expands to global proportions. The dependence of the success of these satellites on reliable hardware and software systems also increases proportionally, as does the corresponding risk of failure of these systems. In the last two years alone, nine spacecraft experienced hardware or software malfunctions which rendered them temporarily unusable but recoverable. As short-term hardware replacement on in-orbit spacecraft is impossible, a flexible software package designed to address and resolve all types of spacecraft anomalies is the ideal means by which to recover the spacecraft.

COTS software packages have the key advantages of flexibility and breadth of functionality to achieve anomaly resolution. Traditional, in-house software packages are generally developed to address normal operations of a particular satellite or system. Due to the budgetary concerns of developing a complete software system specific to a spacecraft or system, satellite companies often do not have the means to incorporate anomaly-resolution tools into their in-house software. COTS systems, which offer the benefit of lower cost, go beyond just meeting the normal operational requirements during nominal space-systems operation. Flexibility and breadth of functionality are vital due to the many types of anomalies which can be encountered and the procedures required for resolution, including:

- $\quad$ Accessing the Space Surveillance Network (SSN)

- Orbit determination

- Orbital lifetime remaining

- Orbit maneuver

- $\quad$ Attitude determination

- $\quad$ Attitude maneuver

- Collision avoidance

- Solar illumination analysis (Power and Thermal)

- Determination of communication availability

- Determination of ground station availability

- Mission capability analysis

Validated and verified COTS systems that have been proven on many satellite missions, those, which address many types of anomalies encountered and perform the 
functions required for resolution, are the ideal tools for these tasks. For the relatively small investment required, typically tens of thousands of dollars, these packages can be, at a minimum, an inexpensive insurance policy. In several cases throughout the past few years, however, COTS packages have been used to rescue billion-dollar satellites. The fast, flexible analysis these software packages afford have already helped save billions of dollars of space systems and will undoubtedly continue to be a vital tool to current and new systems as the proliferation of satellites continues.

\section{References}

1. SpaceVest and KPMG Peat Marwick, State of the Space Industry (KPMG, 1997).

2. Stephen A. Book, Cost-Risk Analysis Seminar - Its Role in Proposal Preparation, System Development, and Program Control (The Aerospace Corporation, 1999).

PAUL L. GRAZIANI is the President and Chief Executive Officer of Analytical Graphics, Inc. (AGI), the producer of the Satellite Tool Kit (STK) ${ }^{\circledR}$ software suite, leading commercialoff-the-shelf software package for the space industry. Mr. Graziani can be reached via phone: +1 610-578-1000; fax: +1 610-578-1001; or e-mail: paul@stk.com. The AGI web site is located at http://www.stk.com.

Satellite Tool Kit is marketed internationally by International Aerospace that can be contacted via phone +44 1908 551105, Fax +44 1908551063 or e-mail: info@intaero.com. 